\title{
Measurement of Local Specimen Temperature under Flowing Gas Ambient in the Environmental Scanning Transmission Electron Microscope (ESTEM) Using Diffraction
}

\author{
Jonathan Winterstein, ${ }^{1}$ Pin Ann Lin $^{1,2}$ and Renu Sharma ${ }^{1}$ \\ 1. Center for Nanoscale Science and Technology, NIST, Gaithersburg, Maryland, USA \\ 2. Institute for Research in Electronics and Applied Physics, University of Maryland, College Park, USA
}

In situ heating experiments in the TEM have been widely used to study many materials phenomena. Among the factors limiting more quantitative analysis of in situ TEM data is a precise knowledge of the sample temperature during a reaction. Going back to the earliest days of in situ and gas-cell TEM experiments accurate measurement of the temperature at the specimen during heating has been an issue. The lack of information about the specimen temperature is particularly problematic for experiments with flowing gases which significantly cool the sample [1]. The use of a thermocouple near the sample cradle can estimate the temperature, but the newer MEMS-based heating holders do not have thermocouples and temperature calibration is performed ex situ in vacuum with a light pyrometer. It is desirable to have a technique for precise, local temperature measurement over a large temperature range that can be applied under flowing gas conditions relevant to ESTEM experiments.

We have used selected area diffraction (SAD) from silver particles deposited on TEM grids to measure lattice expansion as a simple technique for temperature measurement that can be applied on current microscopes without any modification. SAD patterns are acquired and the precise center of diffraction peaks is determined using the plugin Circular Hough Transform from David Mitchell [2]. The shift in diffraction rings is easily observable after alignment as seen in figure 1. The lattice expansion is determined by fitting a Gaussian function to a narrow region of the 311 diffraction peak. For a furnace-type holder, the lattice expansion closely matches literature values for silver for the temperature from the thermocouple [3, 4], at least above $\approx 225^{\circ} \mathrm{C}$, as seen in figure 2a. Statistical analysis of the data indicates an uncertainty in temperature measurement of $\pm 30^{\circ} \mathrm{C}$ at the $95 \%$ confidence level.

Temperature drops were measured under conditions of flowing gas. Figure $2 b$ shows the measured temperature for high vacuum and three different pressures of hydrogen. At a pressure of $135 \mathrm{~Pa}(1.35$ mbar) a temperature drop of almost $300{ }^{\circ} \mathrm{C}$ for a starting temperature of $500{ }^{\circ} \mathrm{C}$ is measured for a furnace-type holder. With flowing hydrogen the thermocouple temperature no longer matches the measured specimen temperature.

The measurements were repeated for a MEMS-based holder. In this case the drop in temperature for flowing hydrogen is slightly smaller. Using the measurements of temperature for flowing hydrogen, estimates of temperature change for other gases at the same pressure can be calculated because of the relatively simple geometry of the MEMS design. Although hydrogen presents a worst-case scenario for temperature change, significant temperature drops are expected for other commonly used gases.

Limitations of the method and alternatives will be discussed.

[1] EP Butler, KF Hale in, "Practical Methods in Electron Microscopy", ed. Audrey Glauert, (North-Holland Pub. Co., New York). 
[2] DRG Mitchell Ultramicroscopy 108 (2008) 367.

[3] I-K Suh, H Ohta, Y Waseda J Mater Sci 23 (1988) 757.

[4] DE Gray (1972) American institute of physics handbook. 3. ed. Coordinating ed.: D.E. Gray. (McGraw-Hill, New York).



Figure 1: A portion of the radial profiles of SAD patterns from silver acquired at different temperature is shown in (a) and two original SAD patterns are shown in (b); the slight shift in diffraction ring diameter is difficult to observe without precise centering and rotational averaging.

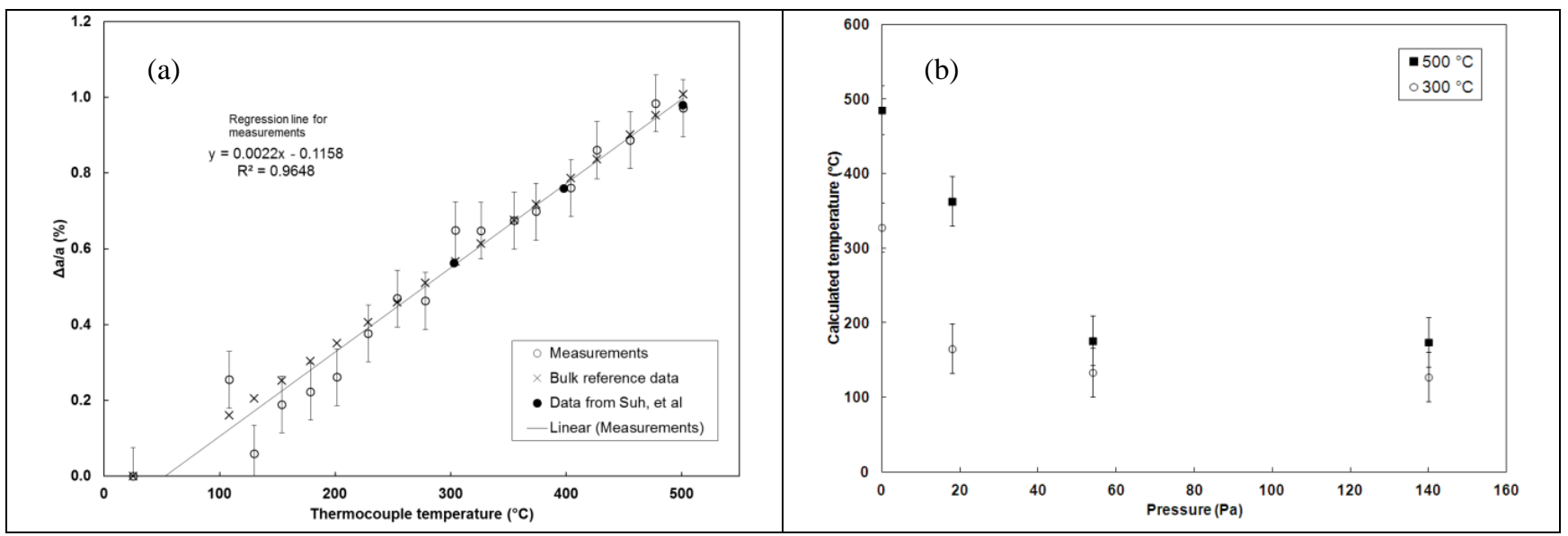

Figure 2: A plot of the measured lattice expansion against the thermocouple temperature for the furnace holder along with lattice expansion values for bulk silver from refs. 3 and 4 (a) and (b) a plot of the change in measured sample temperature for flowing hydrogen at three pressures and for two starting temperatures. 